\title{
MULTISCALE SPARSE APPEARANCE MODELING AND SIMULATION OF PATHOLOGICAL DEFORMATIONS
}

\author{
Rami Zewail and Ahmed Hag-ElSafi \\ Smart Empower Innovation Labs Inc., Canada
}

\begin{abstract}
Machine learning and statistical modeling techniques has drawn much interest within the medical imaging research community. However, clinically-relevant modeling of anatomical structures continues to be a challenging task. This paper presents a novel method for multiscale sparse appearance modeling in medical images with application to simulation of pathological deformations in X-ray images of human spine. The proposed appearance model benefits from the non-linear approximation power of Contourlets and its ability to capture higher order singularities to achieve a sparse representation while preserving the accuracy of the statistical model. Independent Component Analysis is used to extract statistical independent modes of variations from the sparse Contourlet-based domain. The new model is then used to simulate clinically-relevant pathological deformations in radiographic images.
\end{abstract}

Keywords:

Appearance Model, Contourlet, Sparsity, Independent Component Analysis, Pathology Deformations

\section{INTRODUCTION}

Within the field of medical imaging, machine learning and statistical modeling have drawn much attention in a wide range of applications. Examples include: computer-aided diagnosis, segmentation, registration, and surgical planning. However, specificity and generalizability continues to be two major challenges in statistical modeling and learning approaches in medical imaging. This is partly due to noise in the images, complexity of the anatomical structures of interest, and higher resolution of medical images. Lately, in the era of Big Data, this challenge has even escalated where there is an increasing demand for efficient modeling and analyzing of high resolution medical images and large scale of biomedical data in general. As a response to these renewed challenges, concepts of sparse representation and sparse learning have been drawing much attention lately within the medical imaging research community.

In the machine learning paradigm, statistical appearance models fall under the category of Generative models. Generative models are often used in medical imaging to provide constrained solutions for various complex ill-posed problems. Over the last decade, the concept of sparsity of representation and its applications in computer vision has been gaining an increased interest from the research community. In the medical imaging paradigm, sparsity techniques have been used in applications such as image enhancement, segmentation, quantification of diseases. Despite being a promising approach, this continues to be a challenging task due to the complexity of anatomical structures, and scarcity of training sets with ground truth data.

In this paper, we first present a novel method for localized sparse modeling of texture variability in medical images with application to simulation of pathological deformations in medical
$\mathrm{X}$-ray images of human spine. The proposed appearance model is based upon Contourlet transform and Independent Component Analysis (CTICA-AM). Contourlet transform is inherently suited for representation of higher order singularities in medical images. This is due to the multi-scale, directionality and anisotropy of Contourlet basis functions. Localized texture modes of variation are then captured using ICA modeling in the compressed Contourlet domain. The proposed appearance model benefits from the non-linear approximation power of Contourlets to achieve high data reduction rates while preserving the accuracy of the statistical model. This is particularly important in modeling high resolution images.

Next, we present a general framework for simulation of various pathological deformations in $\mathrm{x}$-ray images of human spine. Within the medical imaging community, this is particularly important due to the sacristy of ground truth data. Ground truth data is needed for a number of tasks such as: validation of segmentation and registration algorithms, and improving the training phase in statistical models. The proposed appearance simulation framework makes use of the new proposed sparse appearance model, along with the multi-scale shape model presented in [24].

The rest of the paper is organized as follows: Section 2 covers some of the related work in literature. Section 3.1 presents the details of the proposed Multiscale Sparse Appearance Model. Section 3.2 describes a general image framework for generation of spine X-ray images with simulated pathological deformations. Experiments and results are presented in section 4. Finally, conclusions are drawn in section 5 .

\section{RELATED WORK}

Since the introduction of Active Appearance Models, in [1], modeling of texture appearance in medical images has been predominantly performed using Principle Component Analysis (PCA) basis functions. In order to overcome limitations of Active Appearance Models, a number of researchers suggested different methods to impose locality in appearance modeling. Roberts and Cootes, [2], proposed a method to capture localized texture variations using linked active appearance models. Delac et al. [3], proposed a statistical appearance model using Independent Component Analysis (ICA). The model is used for face recognition. In [4], Zhang et al. presented a method for modeling human faces with parameterized local shape morphing. In [5], Mikkel Stegmann et al. presented a method for sparse modeling of landmark and texture variability using the Orthomax criteria. The model generates localized shape and texture variations. In [6], Wolstenholme and Taylor proposed using Haar-based wavelet compression in Active Appearance Models. A compression ratio of 20:1 was accomplished while maintaining acceptable accuracy of the model. In [7], Stegman and Cootes extended the work in 
[6] by presenting a wavelet enhanced appearance model using biorthogonal CDF 9-7 wavelet basis. In [8], Larsen et al. presented a texture enhanced appearance model using Wedgelets. The Wedgelet representation was shown to be superior to wavelet representation at higher dimensionality reduction rates. In [9], the authors presented an active appearance model based on Gabor wavelets and Principle Component Analysis (PCA). A wavelet appearance representation was used in [10] for pathology classification. In [11], the author used Contourlet Transform for denoising of medical images. All these models relied on Principle component analysis to extract the modes of variations in the appearance model.

A number of authors in the literature have proposed methods for synthesis of simulated data. Abboud et al. [12], proposed a method for facial expression synthesis using PCA-based appearance models. In [13], Seals et al. proposed a method for enlarging the training set using image morphing. In [14], lee et al. presented Polymorph: a method for morphing multiple images. In [15], Hamarneh et al. presented a method for simulation of ground truth validation data via physically- and statistically-based warps. Rose, and Taylor [16], proposed a statistical model for mammographic appearance using steerable decomposition and PCA modeling. In [17], ElSafi et al. proposed a method for statistical simulation of deformations using wavelet Independent Component Analysis. In [18], Xue et al. presented a method for simulating of deformations of MR brain images for validation of Atlas-based segmentation and registration algorithms. In [19], the authors presented a method for prostate segmentation on MRI images based on Active Appearance Model and deep learning. A medical image fusion scheme based on Contourlet Transform and Principle Component Analysis (PCA) bases was proposed in [20].

\section{METHODOLOGY}

The section 3.1 section presents details for the proposed Multiscale Sparse Appearance Model for spine x-ray images. The section 3.2 describes a general image framework for generation of spine $x$-ray images with simulated pathological deformations.

\subsection{SPARSE APPEARANCE MODEL USING CONTOURLETS AND INDEPENDENT COMPONENT ANALYSIS}

This section presents details for the proposed sparse appearance model using contourlet-based representation and independent component analysis (ICA). The new model is also capable of extracting spatially localized texture variations that favours sparsity. The model exploits following concepts: (i) the ability of Contourlets to represent higher order singularities, (ii) the high approximation power of Contourlets, and (iii) the locality of ICA basis. The Fig.1 summarizes the building blocks of the proposed Contourlet enhanced appearance model. Throughout the paper we refer to the new texture appearance model as Contourlet enhanced texture appearance model, or Contourlet-ICA texture appearance model (CTICA-AM).

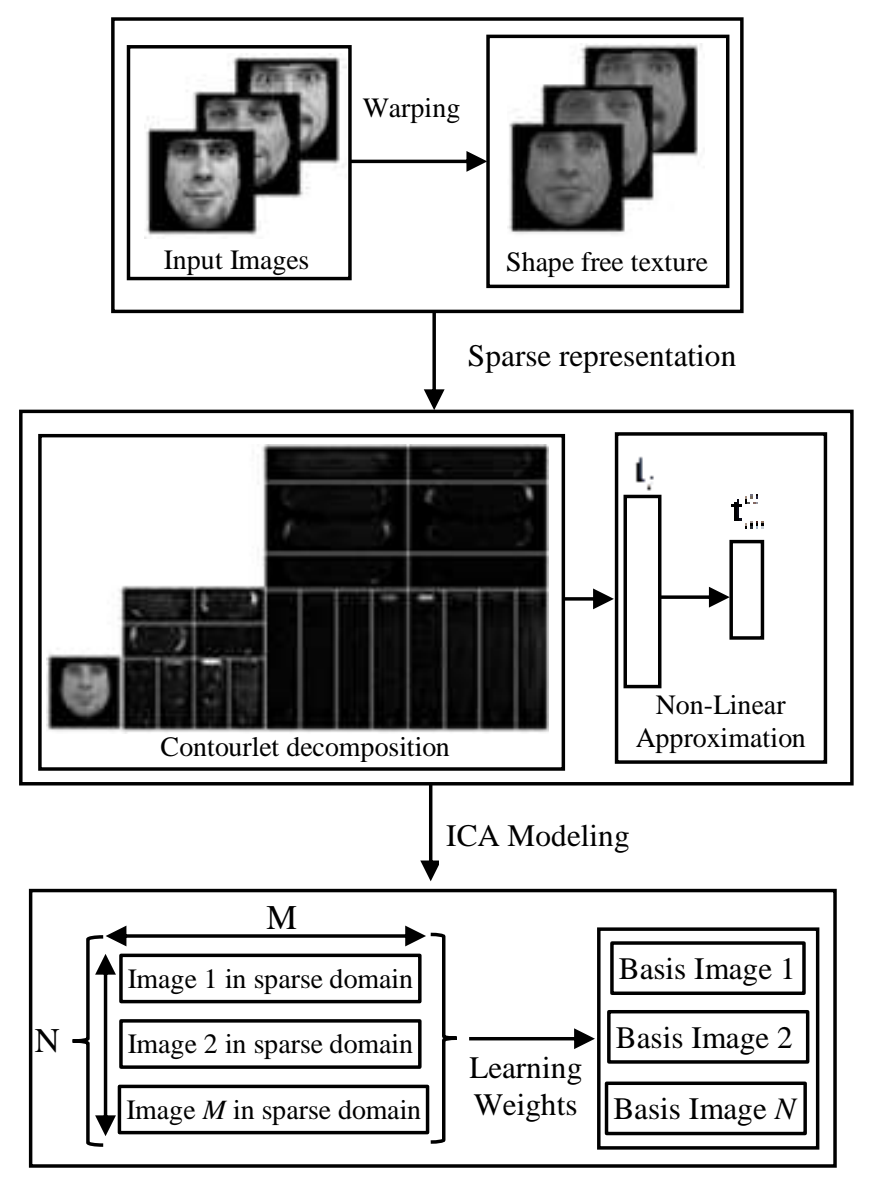

Fig.1. Block diagram for the Contourlet-based Enhanced texture appearance model

\subsubsection{Contourlet Decomposition:}

Multi-resolution transforms try to represent a signal as sparse as possible, i.e. with few coefficients. Contourlets were introduced by Minh Do. And Martin Vetterli as a geometrical second generation wavelets that can deal with the problem of higher order singularities in images [21]. This is attributed to a number of appealing properties for the Contourlet basis; such as: multi-scale, multi-directionality, and anisotropy.

We start with a training set of images, each with $\mathrm{N}$ landmark points marking the structure of interest in the image. Let the $n^{\text {th }}$ shape vector be represented as:

$$
S_{n}=\left[x_{n 1} \ldots \ldots x_{n k}, y_{n 1} \ldots \ldots y_{n k}\right]^{T}
$$

where, $x$ and $y$ are $x$ and $y$-coordinates.

In this work, we use two sets of images to evaluate the proposed texture appearance model. These are namely; sets of face and spine images. The face images were obtained from the IMM face database, [22], and the spine x-ray images were obtained from the National Library of Medicine database [23]. The face images are used to evaluate the proposed method in applications outside the medical imaging paradigm. The IMM face database, [22], is usually used to evaluate appearance models in face recognition applications. To evaluate the performance of the proposed method in medical applications, we use the spine $\mathrm{x}$ ray images from the National Library of Medicine [23]. These $\mathrm{x}$ ray images are used to demonstrate the ability of the proposed 
methodology to simulate more complex pathological deformations.

Given a training set of shape-free texture images, we perform a multi-scale directional image decomposition using the Contourlet transform. We use a 4-level Contourlet transform with $0,4,8$, and 16 directions respectively.

Let $\left\{\mathbf{g}_{i}\right\}_{i=1, \ldots, P}$ be set of vectors of sampled pixel intensities at $L$ locations for the $P$ shape-free images in the training set.

$$
\mathbf{g}_{i}=\left[\mathbf{g}_{i 1}, \mathbf{g}_{i 2}, \mathbf{g}_{i 3}, \ldots, \mathbf{g}_{i L}\right]^{T}
$$

where, $\left\{\mathbf{t}_{i}\right\}_{i=1, \ldots, P}$ is the set of vectors of concatenated Contourlet coefficients that result from the decomposition of the shape-free texture images in the training set.

$$
\mathbf{t}_{i}=C_{T}\left[\mathbf{g}_{\mathrm{i}}\right]
$$

where $C_{T}[\cdot]$ is the contourlet transform operator.

\subsubsection{Non-linear Approximation:}

Next, we exploit the approximation power of Contourlets to tackle the problem of modeling images with high resolution. For a training ensemble of $P$ images, we have a set of vectors of concatenated Contourlet coefficients $\left\{\mathbf{t}_{i}\right\}_{i=1, \ldots, P}$. We select subset of the contourlet coefficients by choosing $\mathrm{m}$ coefficients that best approximate the texture vectors, in terms of mean square error (MSE), while preserving the per-coefficient variance over the training set. Let $\mathbf{C}$ denotes a modified identity matrix that reflects the indices of the coefficients to be retained. Thus, the compressed Contourlet-based representation for images in training set is given by $\left\{\left(\mathbf{t}_{m}^{c t}\right)_{i}\right\}, i=1 . . P$, where:

$$
\left(\mathbf{t}_{m}^{c t}\right)_{i}=\mathbf{C} \mathbf{t}_{i}
$$

\subsubsection{ICA Modelling in Compressed Contourlet Domain:}

Next, we use Independent Component Analysis (ICA) to model texture variability in the compressed Contourlet domain. Traditionally, modeling texture in active appearance models has been performed using Principle Component Analysis (PCA). However, as discussed earlier, pathologies are typically localized not only in shape, but in texture as well. We propose to use ICA to extract spatially localized texture variations that favours sparsity.

We perform the ICA modeling in the compressed sparse Contourlet domain. Applying the ICA on the selected contourlet coefficients has the following advantages: First, it improves the performance of ICA algorithm since projecting the data matrix onto a sparse domain increases the independence of the sources. Second, it increases the robustness to noise and missing information by removing the noisy components through the Contourlet approximation step.

Let $C_{T}$ [ ] be the linear contourlet transform, then the ICA model can be directly re-written in the contourlet domain as:

$$
\begin{aligned}
& C_{T}[X]=A C_{T}[S] \\
& C_{T}[Y]=W C_{T}[X]
\end{aligned}
$$

Then the Contourlet-ICA basis matrix is defined as $\Phi_{C T-I C A}=$ $C_{T}[Y]$, where $C_{T}[Y]$ is the matrix whose rows contain statistically independent basis in the contourlet domain.

Finally, the synthesis of new texture using the Contourlet enhanced appearance model is accomplished by using the learnt Contourlet-ICA model to predict selected contourlet coefficients, and insert mean coefficient values at positions of all truncated coefficients.

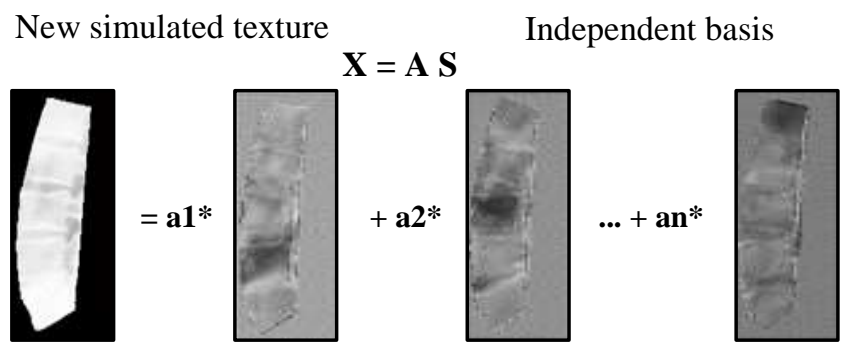

Fig.2. Enhanced texture appearance modeling using ICA basis in Contourlet domain

\subsection{PATHOLOGICAL SIMULATION FRAMEWORK}

This section presents the details for a novel image synthesis framework to generate simulated spine $\mathrm{x}$-ray images with various pathological deformations. The new framework integrates shape and texture generative models proposed earlier in this thesis, along with an example-based pose model. The following presents the details of the proposed framework:

A grey level image $I$ is viewed as a mapping from $R^{2}$ to the range of possible gray levels.

$$
I: R^{2} \rightarrow[0,255]
$$

Let $\left\{I_{i}, S_{i}\right\}, i=1, \ldots, N$ be a set of $N$ images used for building the synthesis framework. $I_{i}$ denotes the input image, $S_{i}$ is a vector of the landmarks that outline the contour of the anatomical structure of interest.

Let $\left\{I_{o}, S_{o}\right\}$ denotes the reference image and the corresponding reference contour.

Let $A_{i}$ denotes the affine transformation that aligns the reference shape $\left(S_{o}\right)$ to the shape $S_{i}$. This is found using Procrustes Alignment. $S_{i}$ and $\left(A_{i}, S_{o}\right)$ has the same in-plane pose.

Let $W_{i j}$ denotes the warping function that maps $\left(I_{i}, S_{i}\right)$ to $\left(I_{j}, S_{j}\right)$

$W_{i j} \cdot I_{i}$ denotes the application of the warping function $W_{i j}$ to $I_{i}$

The warping function is estimated using the piece-wise affine warping method. Piece-wise affine warp is efficient, and allows for inverse warping, hence it can be used for synthesis of realistic images.

An image $I$ is characterized by three components; these are:

- The pose of the structure of interest in the image.

- The texture component of the image which determines the appearance of the structure of interest.

- The shape component which outlines the contour of the structure of interest.

Using the input training set of images $\left\{I_{i}, S_{i}\right\}, i=1, \ldots, N$, we learn the variability in each of these three components. These are referred to as: pose, shape and texture spaces. In each of these $\mathrm{N}$ 1 dimensional spaces, the image is viewed as a point whose coordinates constitutes a parameter vector. Let $\boldsymbol{\alpha}_{\mathbf{P}}=\left[\alpha_{P_{1}}, \alpha_{P_{2}}, \ldots\right.$, $\alpha_{P N}$ ] represent the pose-parameter vector. $\boldsymbol{\alpha}_{\mathbf{S}}, \boldsymbol{\alpha}_{\mathrm{T}}$ represent the shape and texture parameter vector respectively.

We learn the shape space using the multi-scale shape model presented in [24]. The generative Contourlet enhanced 
appearance model presented in section 3 is used to model the texture space. An example-based pose model is used to represent variations in translation and scaling within the input data set.

\subsubsection{Multi-Scale Shape Model:}

We model variations in the shape of anatomical structure of interest using the multi-shape model presented in [24]. Using wavelet-packets and Independent Component Analysis, the model extracts multi-scale localized modes of deformations. Due to the locality of the basis functions, the model is then used to selectively impose various pathology-related deformations, such as: disc-narrowing, osteophytes and vertebral fractures.

We start with a training set of landmarks that outline the structure of interest in the x-ray images. Let $\Phi_{\text {wICA }}$ be the matrix of learnt multiscale Contourlet-ICA basis set. The multi-scale generative shape model is represented as follows:

$$
S=S_{o}+\alpha_{\mathrm{s}} \boldsymbol{\Phi}_{\text {wiCA }}
$$

A new shape $(S)$ is represented as a sum of reference shape $\left(S_{o}\right)$ and weighted sum of the multi-scale basis functions. By controlling the weighting vector $\left(\boldsymbol{\alpha}_{\mathrm{s}}\right)$ one can impose certain localized deformations to the reference shape. Note that the multiscale shape model includes also inverse transformation from wavelet-packet domain to spatial domain. However, this step is eliminated from Eq.(9) for clarity.

\subsubsection{Texture Appearance Model:}

A new texture vector $(T)$ is generated by summation of the reference texture vector $\left(T_{o}\right)$ and weighted sum of Contourlet-ICA texture basis set $\left(\Phi_{\text {CTICA }}\right)$.

$$
T=T_{o}+\alpha_{\mathrm{T}} \boldsymbol{\Phi}_{\text {CTICA }}
$$

where, $\boldsymbol{\alpha}_{\mathrm{T}}$ is vector of weights used to introduce new appearance to the image.

\subsubsection{Example-based Pose Model:}

We simulate realistic poses of the spine in x-rays using an example-based pose model. This model is used to generate new images at varying pose. Given a training set of lumbar spine images with various poses $\left(\left\{I_{i}, S_{i}\right\}, i=1, \ldots, N\right)$, we select a number of proto-type poses.

Using the proto-type pose examples, we interpolate among the pose space to generate new poses for the spine. Let $\left\{\tilde{S}_{o}^{i}, i=1 . . M\right\}$ be the set of proto-type poses for the reference shape obtained by aligning the reference shape with the set of selected prototypes poses, i.e. $\tilde{S}_{o}^{i}$ and $S_{i}$ have the same pose.

$$
\tilde{S}_{o}^{i}=A_{i} S_{o}
$$

We then use interpolation techniques to synthesis a new pose within the $M-1$ dimensional pose space. A new pose for the reference shape $\left(S_{o, p}\right)$ is then defined as weighted sum of the selected proto-type poses.

$$
S_{o, p}=\sum_{i} \alpha_{p} \tilde{S}_{o}^{i}
$$

The Fig. 3 shows example of a two-dimensional pose space using four proto-type poses $\left\{\tilde{S}_{o}^{i}, i=1,2,3,4\right\}$. We use bi-linear interpolation among the four proto-type pose examples to synthesize a new pose for the reference shape $\left(S_{o, p}\right)$ at the point with coordinates $(x, y)$.

$$
S_{o, p}=\sum_{i=1}^{4} \boldsymbol{\alpha}_{\mathbf{p}}(i) \tilde{S}_{o}^{i}
$$

where,

$$
\begin{aligned}
& \boldsymbol{\alpha}_{\mathbf{p}}(1)=(1-x)(1-y), \boldsymbol{\alpha}_{\mathbf{p}}(2)=(x)(1-y), \\
& \boldsymbol{\alpha}_{\mathbf{p}}(3)=(1-x)(y), \boldsymbol{\alpha}_{\mathbf{p}}(4)=(x y), \text { and } x, y \in[0,1] .
\end{aligned}
$$

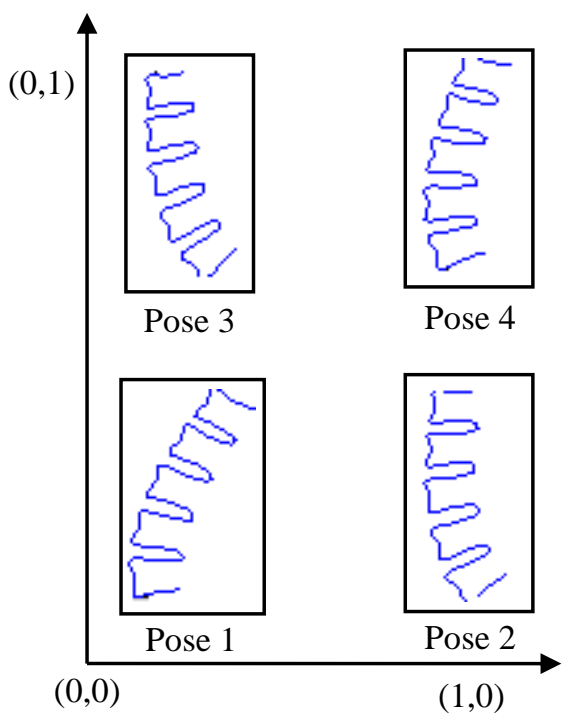

Fig.3. Example of two-dimensional pose space using four prototype poses 


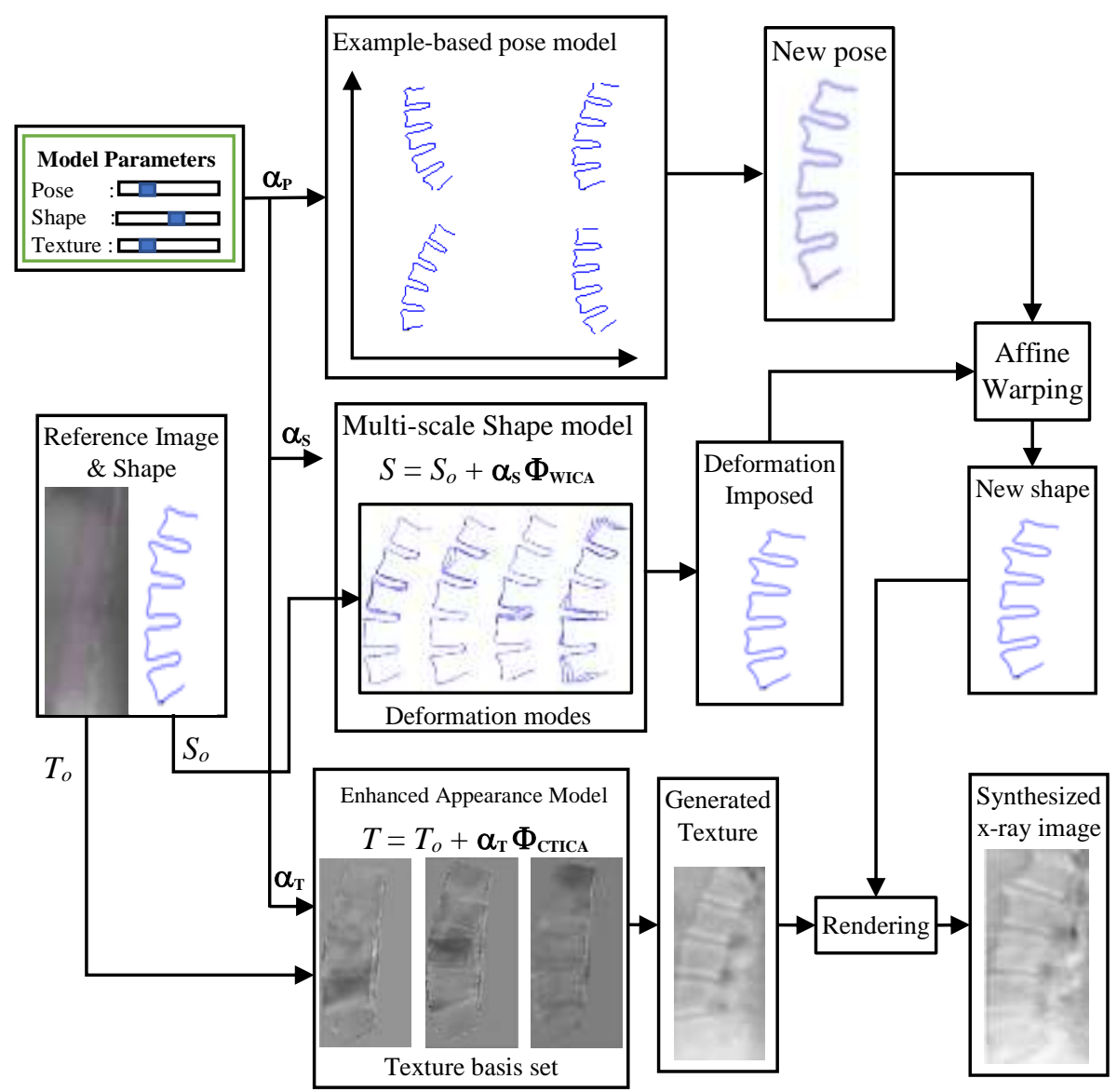

Fig.4. Block diagram of proposed spine x-ray image synthesis framework

The Fig.4 illustrates the overall image synthesis framework. The following summarizes the steps for synthesis of an $\mathrm{x}$-ray image with certain imposed pathology.

i. Starting with the reference shape $\left(S_{o}\right)$, we use the basis of the multi-scale shape model to impose the desired pathological deformation. The outcome is a new shape $\left(S_{n}\right)$. This is shown in Eq.(8).

ii. A new texture appearance $\left(T_{n}\right)$ is then generated using the basis set of the Contourlet enhanced appearance model $\left(\Phi_{\text {Cтіс⿱ }}\right)$, and the texture parameter vector $\left(\boldsymbol{\alpha}_{\mathrm{T}}\right)$. This is shown in Eq.(9).

iii. We use the pose parameter vector, $\boldsymbol{\alpha}_{\mathbf{p}}$, to generate a new pose for the reference shape $\left(S_{o, p}\right)$. Find the transformation matrix $\left(A_{p}\right)$ that maps $S_{o}$ to $S_{o, p}$.

iv. Align the new shape $\left(S_{n}\right)$ generated in (i) so that it has same pose as $\left(S_{o, p}\right)$.

$$
S_{n, p}=A_{p} S_{n}
$$

$S_{n, p}$ represents the new shape with a new pose.

v. A new image is finally synthesized by rendering the new shape-free texture appearance obtained in (ii) to the new shape vector $S_{n, p}$.

$$
I_{n}=W\left(T_{n}, S_{n, p}\right)
$$

where $W()$ is the operator for the warping function.

\section{EXPERIMENTS AND RESULTS}

This section presents the details for the experiments conducted to evaluate the methods presented in this paper. We use two datasets of images: First, a dataset of 30 images of lumbar spine $\mathrm{X}$-rays with their corresponding ground truth segmentation [23]. Second, a database of 40 images of faces of different individuals with various expressions [22].

\subsection{EVALUATION OF ENHANCED TEXTURE APPEARANCE MODEL}

This section presents the experiments and results to evaluate the performance of the proposed Contourlet enhanced texture appearance model.

\subsubsection{Approximation Power:}

First, we assess the approximation power of contourlet for both the spine x-ray images and the face images. Results are compared to the work of Stegman et al. [7, 8] where authors suggested an enhanced appearance model based upon wavelets.

The Table.1 shows the SNR for contourlet-based representation of the spine $\mathrm{X}$-ray images versus wavelet-based representation at various reduction ratios. In general, the contourlet-based representation tends to achieve higher SNR, especially at higher reduction ratios. At reduction ratio of 1:40, the contourlet-based representation showed an increase in SNR of $11.9 \%$, compared to wavelet-based representation. As the reduction ratio increases to $1: 200$, the contourlet-based 
representation shows an increase in the SNR of $86.3 \%$ over the wavelet-based representation. This agrees with the results obtained from the visual inspection of the reconstructed images, as shown in Fig.5. Even at reduction ratios of 1:200, the contourlet representation appears to better capture the details of the image, as opposed to the wavelet representation which deteriorates quickly at higher reduction rates.

Table.1. Performance of Contourlets versus wavelets in compression of spine texture space

\begin{tabular}{|c|c|c|c|c|}
\hline $\begin{array}{c}\text { Reduction } \\
\text { ratio }\end{array}$ & $\mathbf{1 : 2 0 0}$ & $\mathbf{1 : 1 2 5}$ & $\mathbf{1 : 1 0 0}$ & $\mathbf{1 : 4 0}$ \\
\hline $\begin{array}{c}\text { SNR for } \\
\text { Contourlet- } \\
\text { based model }\end{array}$ & $10.66 \mathrm{~dB}$ & $12.91 \mathrm{~dB}$ & $13.04 \mathrm{~dB}$ & $14.85 \mathrm{~dB}$ \\
\hline $\begin{array}{c}\text { SNR for } \\
\text { Wavelet-based } \\
\text { model }\end{array}$ & $5.72 \mathrm{~dB}$ & $7.41 \mathrm{~dB}$ & $10.16 \mathrm{~dB}$ & $13.26 \mathrm{~dB}$ \\
\hline
\end{tabular}

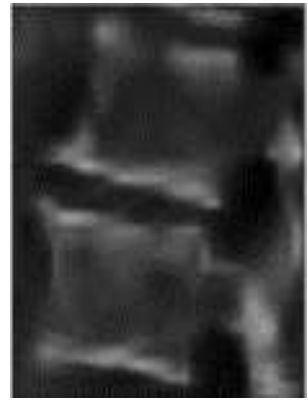

(a)

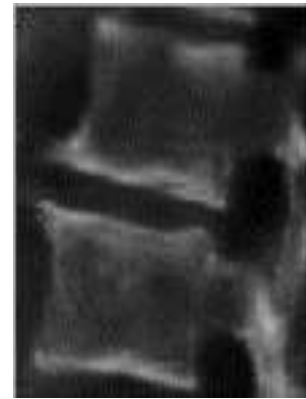

(c)

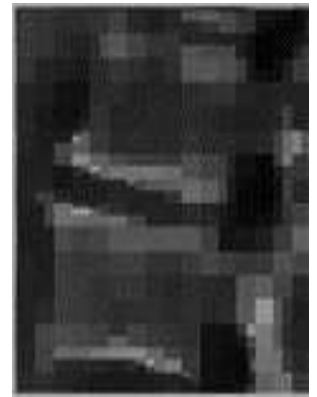

(e)

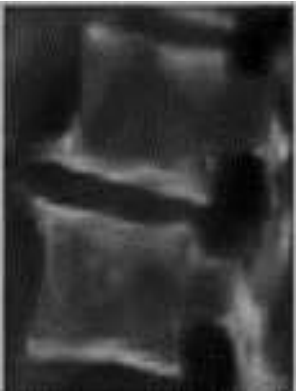

(b)

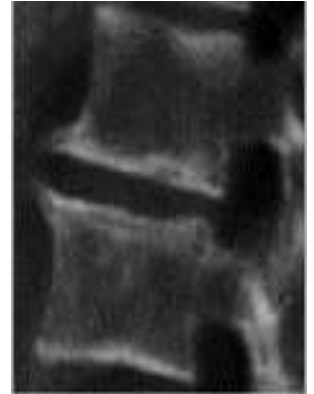

(d)

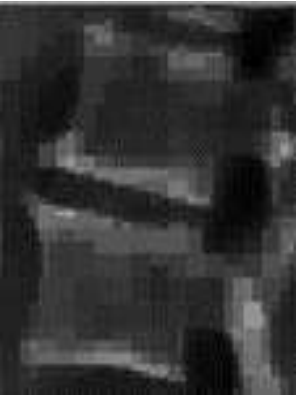

(f)

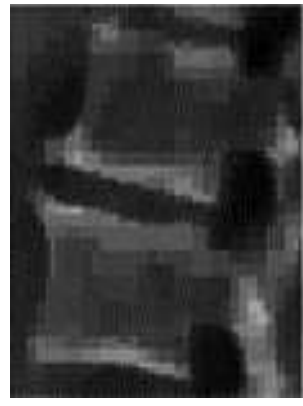

$(\mathrm{g})$

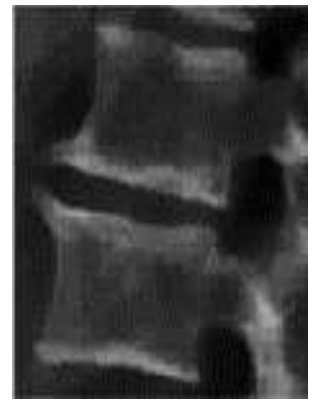

(h)
Fig.5. Non-linear approximation of spine x-ray image using Contourlets (Top row), and (Wavelets). Reduction Ratios: (a)(e) 1: 200 (b)(f) 1:125 (c)(g) 1:100, (d)(h) 1:40

\subsubsection{Locality of Basis:}

The Fig. 6 compares the basis sets for the proposed texture enhanced appearance model (CTICA-AM), and the PCA-based basis set used in the Active Appearance Model (AAM). It is evident that the new model produces localized basis functions, as opposed to the global PCA basis. This property is exploited to impose localized texture variations. The Fig.9 shows examples of face images that are generated using new Contourlet-ICA texture appearance model. Using the localized basis functions, we are able to selectively impose localized deformations.

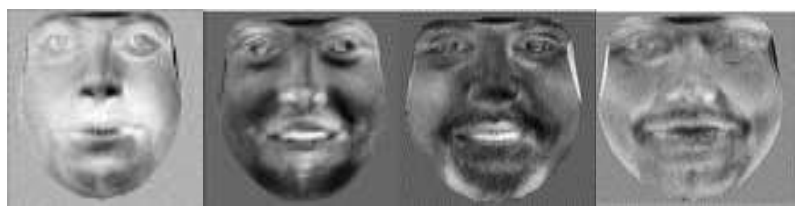

(a)

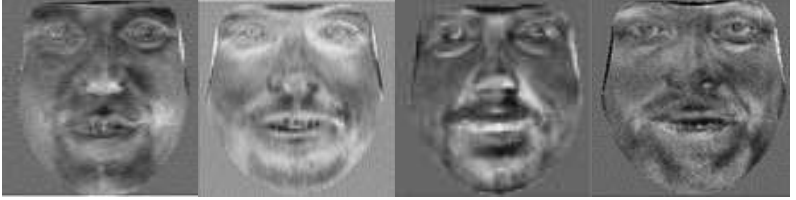

(b)

Fig.6. Examples of basis sets for different texture appearance models. (a) PCA-based Active Appearance Model,

(b) Contourlet-ICA basis of the new enhanced appearance model

\subsubsection{Texture Reconstruction:}

We study the ability of the proposed appearance model to reconstruct un-seen texture appearance examples using limited size of training set. Leave-one-out experiment is conducted where the appearance model is trained using N-1 training images, and the left-over image is used for testing. This is repeated for all the $\mathrm{N}$ images in the training set. The average reconstruction error (also referred to as generalization error) is then calculated as the mean intensity error per pixel (for a 256 gray level image). This is repeated for $\mathrm{N}=\left[\begin{array}{llll}10 & 20 & 30 & 40\end{array}\right]$. The Fig.7 compares the performance of the proposed enhanced appearance model (CTICA-AM) compared to Cootes' Active Appearance Model (PCA-AAM). The new texture appearance model is shown to achieve lower reconstruction error for different sizes of the training set. Using a training set of 10 images, the average reconstruction error of the CTICA-AM model has decreased by 
$12.89 \%$ compared to AAM. On average, the proposed ContourletICA appearance model achieves a decrease of $17.62 \%$ in the texture reconstruction error compared to AAM model.

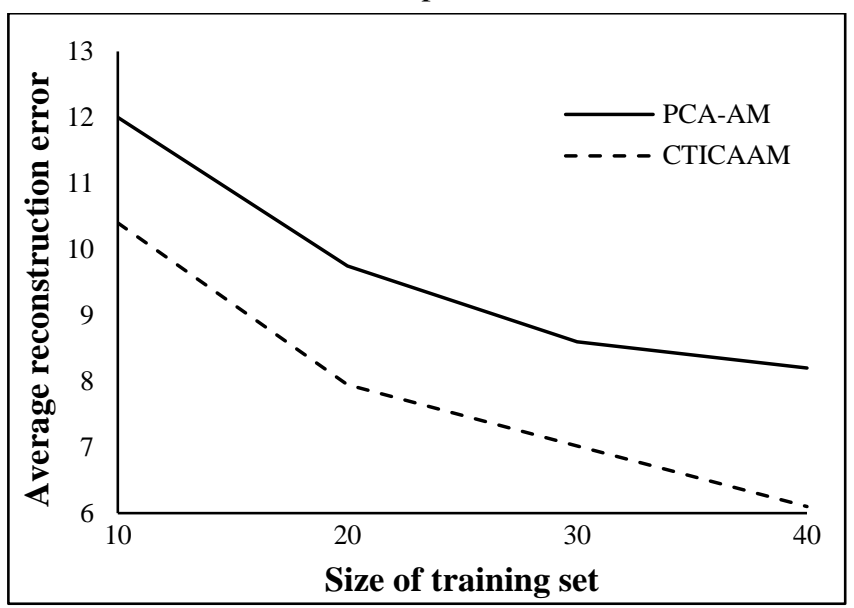

Fig.7. Reconstruction error of the new texture appearance model for different sizes of training sets

\subsection{EVALUATION OF THE PATHOLOGICAL DEFORMATIONS SIMULATION FRAMEWORK}

The Fig. 8 shows examples of synthesized x-ray images where various localized pathology-related shape deformations were imposed. The Fig.8 (h) shows example of disc narrowing between the L1 and L2 vertebrae. The Fig.8(b) and Fig.8(c) shows signs of osteophytes pathology developed for L2 vertebra. The Fig.8(f) shows fractures being imposed on L4 vertebra. The Fig.9 shows examples of synthesized $x$-ray images with localized variations in texture appearance while the shape and pose are kept constant.

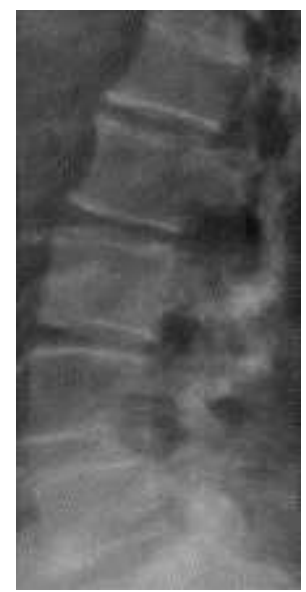

(a)

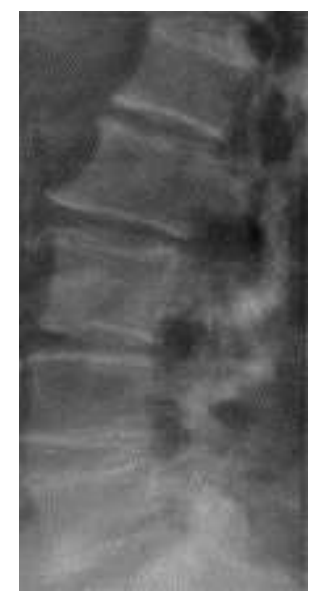

(b)

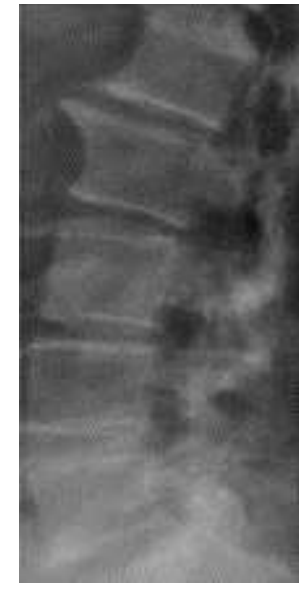

(c)

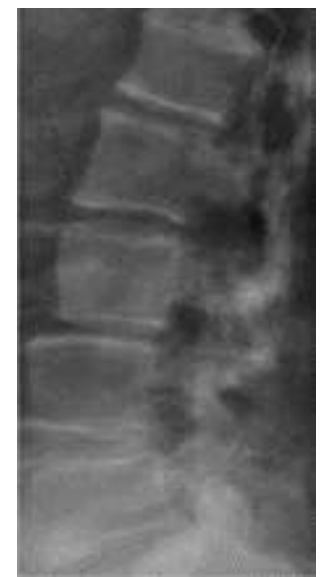

(e)

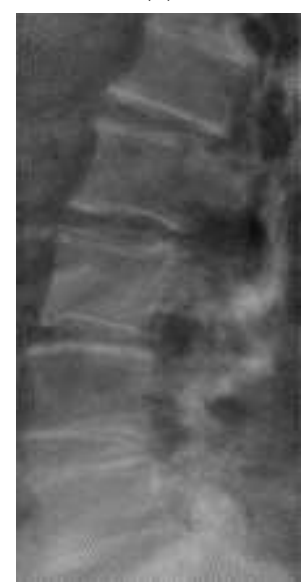

(g)

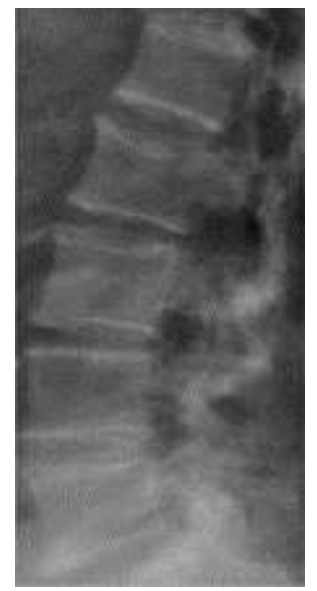

(d)

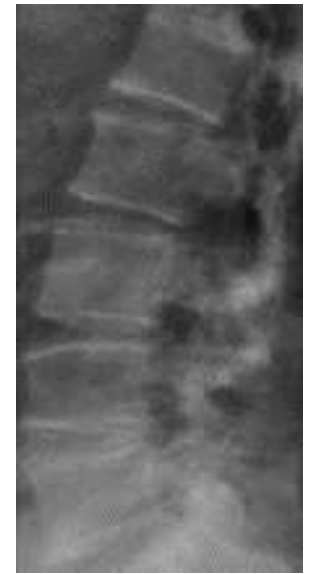

(f)

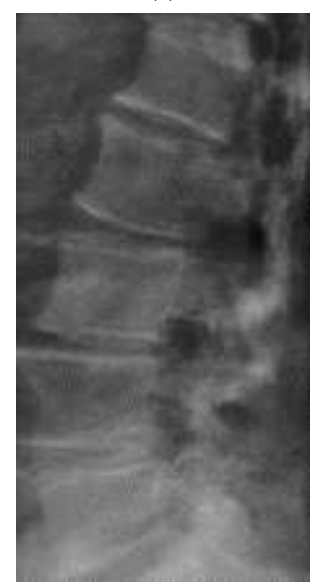

(h)
Fig.8. Examples of simulated x-ray spine x-ray images with various localized shape deformations imposed while the texture appearance and pose are kept constant 


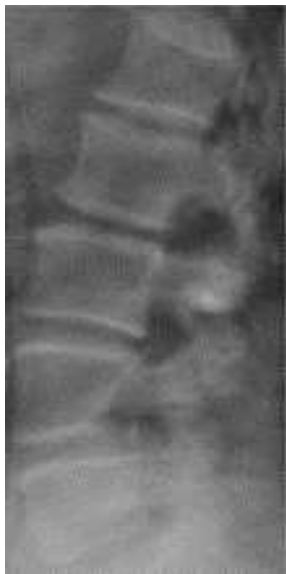

(a)

(c)

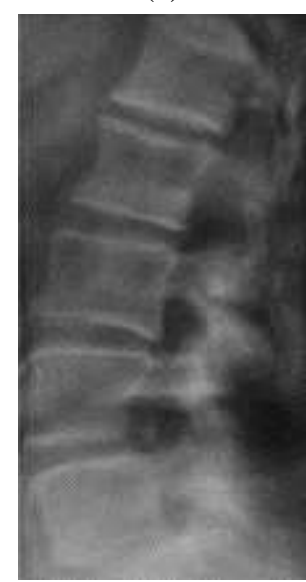

(e)

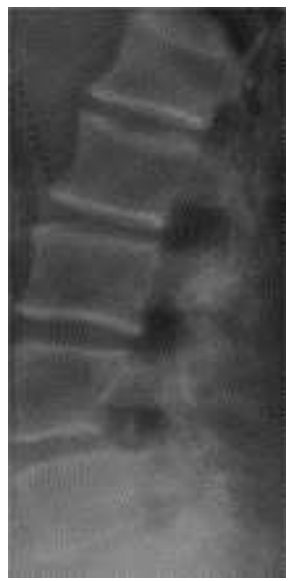

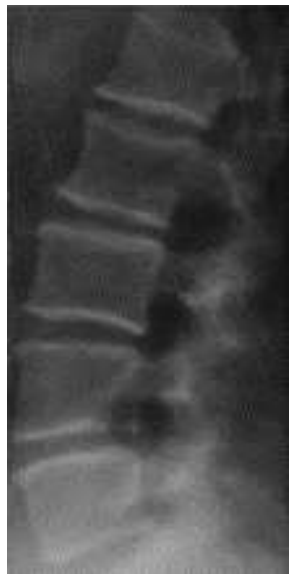

(b)

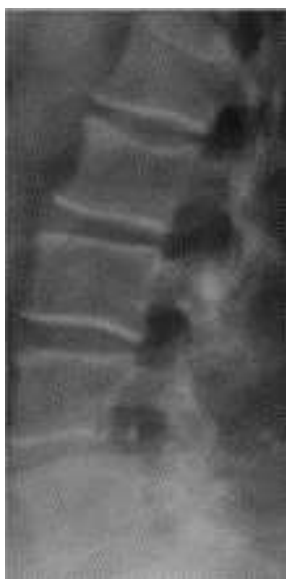

(d)

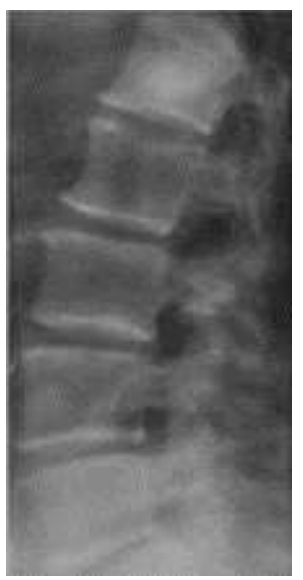

(f)

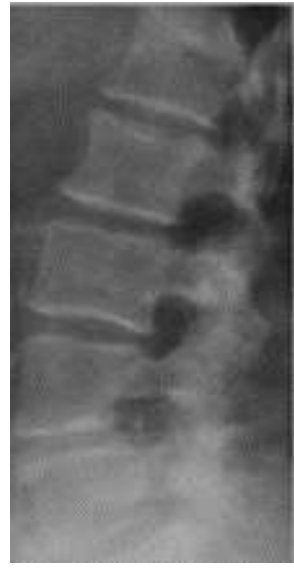

(g)

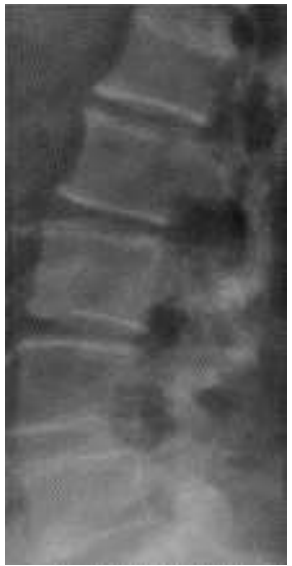

(h)
Fig.9. Examples of simulated x-ray spine images with different texture variations imposed while pose and shape are kept constant

By varying the model parameters $\left(\alpha_{P}, \alpha_{S}, \alpha_{T}\right)$, we can simulate photo-realistic x-ray images with different degrees of pose, shape and texture variations. The outcome is a set of synthesized images with the corresponding ground truth segmentation. The Fig.10 shows examples of the generated images and their ground-truth segmentation. This way it is possible create an enlarged training set with desired shape and texture deformations.

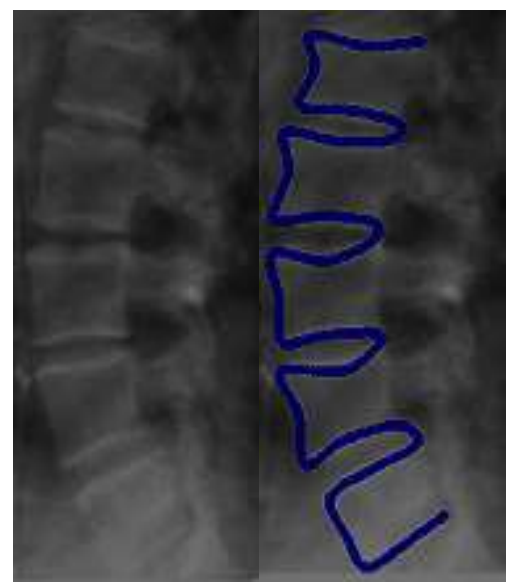

(a)

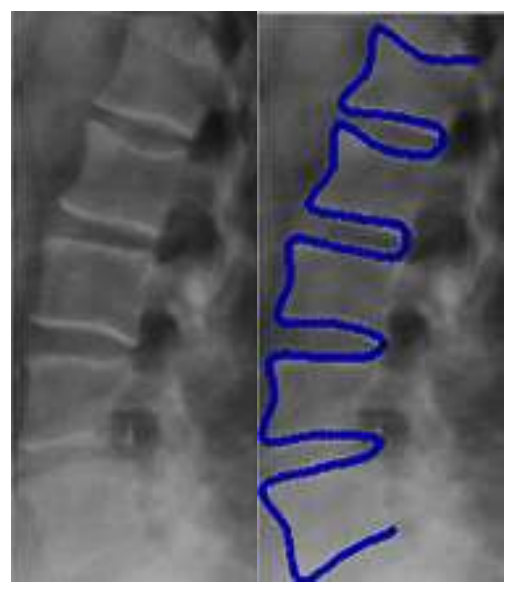

(b) 


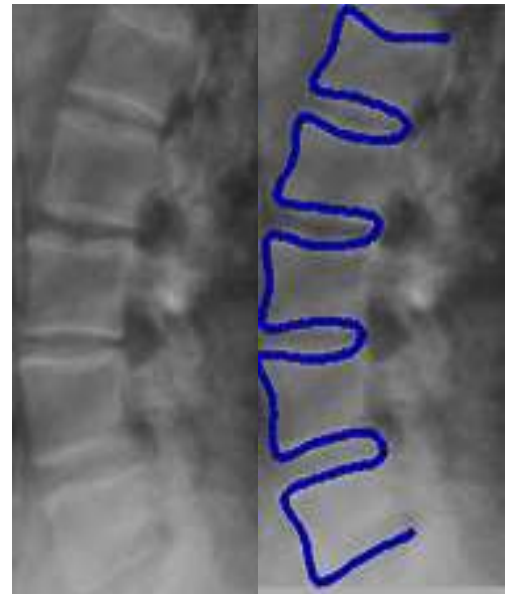

(c)

Fig.10. Examples of simulated ground truth spine $\mathrm{x}$-ray images with the corresponding segmentation

\section{SUMMARY AND CONCLUSION}

In this paper, we tackled challenges related to accurate appearance modeling and sacristy of training data. We presented a new sparse texture appearance model (CT-AM) based upon Contourlet Transform and Independent Component Analysis (ICA). The new model benefits from the non-linear approximation power of Contourlets to achieve high reduction rates, and the Independent Component Analysis (ICA) to capture localized pathology-related texture variations. The new texture Contourlet-enhanced Appearance Model (CT-AM) is shown to outperform related wavelet-based appearance models, especially at higher reduction rates. We also describe a general framework that integrates developed shape and texture models in a unified framework for synthesis of photo-realistic pathological x-ray images. Experiments were conducted to evaluate the performance of the Contourlet enhanced texture model, and the pathology deformation simulation framework. Using human spine $\mathrm{x}$-ray images, the proposed model was shown to be capable of modeling complex localized pathology-related deformations in medical images. In addition to suitability for medical image analysis tasks, the proposed model showed promising results in modeling localized variances in face images. This further illustrates the suitability of the model in a wide range of applications related to face detection and recognition.

\section{ACKNOWLEDGEMENT}

The authors wish to acknowledge the US. National Library of Medicine for making the digital $\mathrm{x}$-ray images available.

\section{REFERENCES}

[1] T.F. Cootes, G.J. Edwards and C.J. Taylor, "Active Appearance Models", IEEE Transactions on Pattern Analysis and Machine Intelligence, Vol. 23, No. 6, pp. 681685, 2001.

[2] M. Roberts, T. Cootes and J. Adams, "Automatic Segmentation of Lumbar Vertebrae on Digitised Radiographs using Linked Active Appearance Models",
Proceedings of Medical Image Understanding and Analysis, Vol. 2, pp. 120-124, 2006.

[3] K. Delac, M. Grgic and P. Liatsis, "Appearance-based Statistical methods for Face Recognition", Proceedings of $47^{\text {th }}$ International Symposium Focus on Multimedia Systems and Applications, pp. 151-158, 2005.

[4] X. Xu, C. Zhang and T. Huang, "Active Morphable Model: An Efficient Method for Face Analysis", Proceedings of $6^{\text {th }}$ IEEE International Conference on Automatic Face and Gesture Recognition, pp. 837-842, 2004.

[5] Mikkel B. Stegmann, Karl Sjostrand and Rasmus Larsen, "Sparse Modeling of Landmark and Texture Variability using the Orthomax Criterion", Proceedings of International Symposium on Medical Imaging, Vol. 6144, pp. 1-12, 2006.

[6] C. Wolstenholme and C. Taylor, "Wavelet Compression of Active Appearance Models", Proceedings of $2^{\text {nd }}$ International Conference on Medical Image Computing and Computer-Assisted Intervention, pp. 544-554, 1999.

[7] M.B. Stegmann, S. Forchhammer and T.F. Cootes, "Wavelet Enhanced Appearance Modelling", Proceedings of Symposium on Medical Imaging, Vol. 5370, pp. 1823-1832, 2004.

[8] R. Larsen, M.B. Stegmann, S. Darkner, S. Forchhammer, T.F. Cootes and B.K. Ersboll, "Texture Enhanced Appearance Models", Computer Vision and Image Understanding, Vol. 106, No. 1, pp. 20-30, 2007.

[9] Ki Gia Quach, Chi Nhann Duong, Khou Luu and Bac Le, "Gabor Wavelet-based Appearance Models", Proceedings of International Conference of Computing and Communication Technologies, Research, Innovation, and Vision for the Future, pp. 1-6, 2012.

[10] Qiang Zhang, Abhir Bhalerao, Caron Parsons, Emma Helm and Charles Hutchinson, "Wavelet Appearance Pyramids for Landmark Detection and Pathology Classification: Application to Lumbar Spinal Stenosis", Proceedings of International Conference on Medical Image Computing and Computer-Assisted Intervention, pp. 274-282, 2016.

[11] Abbas Hanon Hassin Al Asadi, "Contourlet Transform based Medical Image Denoising", International Journal of Image Processing, Vol. 9, No. 1, pp. 22-31, 2015.

[12] B. Abboud, F. Davoine, and M. Dang, "Facial Expression Recognition and Synthesis based on An Appearance Model”, Signal Processing: Image Communication, Vol. 19, No. 8, pp. 723-740, 2004.

[13] W. Seales and C. Yaun, "Improved Image Classification using Morphing", Proceedings of $3^{\text {rd }}$ Asian Conference on Computer Vision, pp. 233-240, 1998.

[14] S. Wolberg and G. Shin, "Polymorph: Morphing among Multiple Images", IEEE Computer Graphics and Applications, Vol. 18, No. 1, pp. 58-71, 1998.

[15] Ghassan Hamarneh, Preet Jassi and Lisa Tang, "Simulation of Ground Truth Validation Data via physically-and Statistically-based Warps", International Conference on Medical Image Computing and Computer-Assisted Intervention, pp. 459-467, 2008.

[16] C.J. Rose and C.J. Taylor, "A Statistical Model of Texture for Medical Image Synthesis and Analysis", Proceedings of Medical Image Understanding and Analysis, pp. 1-4, 2003.

[17] Ahmed Elsafi, Rami Zewail, and Nelson Durdle, "Statistical Simulation of Deformations using Wavelet Independent 
Component Analysis", Proceedings of Symposium on Security and Defence Visual Information Processing, Vol. 6978, pp. 1-8, 2008.

[18] Z. Xue, D. Shen, B. Karacali, J. Stern, D. Rottenberg, and C. Davatzikos, "Simulating Deformations of MR Brain Images for Validation of Atlas-based Segmentation and Registration Algorithms", Neuroimage, Vol. 33, No. 3, pp. 855-866, 2006.

[19] Ruida Cheng et al., "Active Appearance Model and Deep Learning for more Accurate Prostate Segmentation on MRI", Proceedings of Medical Imaging, pp. 1-9, 2016.

[20] R. Nithya and S.Elayaraja, "Medical Image Fusion Schemes using Contourlet Transform and PCA Bases", Asian Journal of Electronics Sciences, Vol. 4, No. 1, pp. 27-33, 2015.
[21] M.N. Do and M. Vetterli, "Contourlets in Beyond Wavelets", Academic Press, 2003.

[22] M. Nordstom et al., "The IMM Face Database: An Annotated Dataset of 240 Face Images, Informatics and Mathematical Modelling”, Technical University of Denmark, 2004.

[23] L. Long, S. Antani and G. Thoma, "Image informatics at a National Research Center", Computerized Medical Imaging and Graphics, Vol. 29, No. 2-3, pp. 171-193, 2005.

[24] Rami Zewail, Ahmed ElSafi and Nelson Durdle, "Vertebral segmentation using Contourlet-based Salient Matching and Localized Multi-Scale Shape Prior", Proceedings of Medical Imaging, pp. 1-9, 2009. 